Article Citation Format

O.E. Taylor, \& O.M Ata-ebirieng (2018): A Hybrid Agent-Based Network Monitoring ToolJournal of Digital Innovations \& Contemp Res. In Sc., Eng \& Tech. Vol. 6, No. 4. Pp 49-56

\begin{tabular}{|r|}
\hline Article Progress Time Stamps \\
Article Type: Research Article \\
Manuscript Received: $27^{\text {th }}$ Sept. 2018 \\
Review Type: Blind \\
Final Acceptance:: $29^{\text {th }}$ Nov, 2018 \\
DOI Prefix: 10.22624 \\
\hline
\end{tabular}

\title{
A Hybrid Agent-Based Network Monitoring Tool
}

\author{
O.E. Taylor, \& O.M. Ata-ebirieng \\ Department of Computer Science \\ Rivers State University of Science and Technology \\ Port Harcourt, Nigeria. \\ E-mail: tayonate@yahoo.com, mikedecomputerist@gmail.com
}

\begin{abstract}
The purpose of this paper is to develop a responsive Agent based network monitoring system designed to reduce the time taken by network administrators to initiate monitoring of network nodes and making any changes and updates on the monitoring parameters. The Simple Network Management Protocol (SNMP) is the most extensively used network monitoring tool but it is limited by its centralized client server and static paradigm. The approach is Ad-hoc, centralized and relies on a limited set of capabilities at network nodes. In this paradigm, the system does not auto respond to changes and modifications in the network as hard coding is required to make the necessary changes. Our approach uses an agent system that provides a solution that works by reducing the time taken to initiate monitoring in network parameters by introducing intelligence to the system. The results of the study showed that the agent or multi-agent system optimizes efficiency in monitoring network parameters and the comparison between the network monitoring conventional system and that of the agent based system in terms of response time.
\end{abstract}

Keywords: Agent, Agent System, SNMP, JADE

\section{INTRODUCTION}

Network management (monitoring) essentially involves monitoring and controlling the devices connected in a network by collecting and analyzing data from the device (Wesley, 1999). The most commonly used internet based protocol for gathering information about managed network nodes is the simple network management protocol (SNMP) which is mainly limited due to the client/server centralization. The centralized nature of this method is due to its reliance on limited set of services embedded at the network nodes as I while the management of these nodes is carried out at the central network management station. The challenge of increased heterogeneous computing has made network monitoring difficult and complex to deal with; hence a better way of approach to network monitoring can be actualized through the use of mobile agents. Agent-based technology offers a decentralized and completely new paradigm where intelligent agents can be used to perform a wide variety of functions such as: information gathering, sensing, reasoning and collaboration with other agents. 
In an agent based system, the software agent with adequate intelligence and autonomy can either work independently or collaborate with other software agents to attain its goal(s) in a specified environment.

With the current development, different sectors such as the banking sector and the deploying of automated teller machine (ATM), the mobile companies delivering SMS services, money transfer service, social networking and all other ways of information flow is highly growing. The relationship of moving management logic close to the data it requires is becoming highly desirable in monitoring architecture. An agent-based system for network monitoring can monitor and control network devices on site and consequently save the manager's capacity and bandwidth and also to reduce burden on network administrators and resources. Software technology is an emerging and promising technology which attracts great interest in recent years, research and development is currently ongoing in the software agent domain. This study was intended to develop using a model, the use of an agent based system that provide several benefits to network management (monitoring) including; distribution of management work load, adaptability, responsiveness, flexibility, intelligence and customization using the JADE Agent platform.

\section{ORIGIN OF AGENT BASED TECHNOLOGY}

The true concept of agent technology originated from artificial intelligence; (Jacques, 1999) and (Woldridge, 2012). Thus, the roots of the concept date back to the 1950's when artificial intelligence (AI) was born, software agents are secret software detectives that provides a general computing platform for executing task like information gathering, information filtering and searching, online shopping, etc. Software agent can exist as sole independent entity or in collaboration with other agents in the same environment to perform specified tasks, in such case can be referred to as multi-agent system. Network monitoring system is type of system that constantly monitors and collect relevant information on a computer network for slow or failing components as well as notifies the network administrator (via email, SMS or other alarms) in case of outages or other network related issues. Network monitoring is part of network management. To fully understand the concept of network monitoring to network management, you can think of it as paying a visit to your doctor for an annual check-up. The doctor uses his/her experience to monitor the performance of various internal organs and parts of your body, during the examination; while you are being monitored, specific data is collected to ascertain your health status.

With network monitoring, the same type of activity is being tracked as data passes through network servers and other components. The system then generates the results of specific activities that have occurred during the monitoring process, monitoring involves server performance, switch and router performance, bandwidth usage and application performance, etc. it is also important to mention that network monitoring is not the same as IDS (intrusion detection system) as the two processes are very different. Network monitoring helps keep track of network health status, bandwidth usage, software updates, etc. IDS is focused solely on security and specifically designed to identify a network breach by an unauthorized user and utilizes a variety of methods to identify a hacking system.

\subsection{Network monitoring software}

Network monitoring software can keep an eye on network traffic and bandwidth usage. It can check for crucial network statistics and network components such as: switches, routers, servers, workstations, (i.e. if they are up or down). Network managers usually set thresholds for acceptable performance, and if the software finds low throughput, high error rates, unavailable devices or slow response time; notification(s) is sent. Some of the devices that network monitoring software can track are email servers, network traffic, DNS servers, storage devices, etc. It can check on and detect both availability and response time. Network monitoring applications differ in their ease of use. 
While some offer only a command line interface or a graphical user interface (GUI) to manage both setting alerts and viewing results. Sometimes the interface uses a proprietary program running on a client on the local network. Many network monitoring tools provide a web interface to manage the application. Some even provide a mobile device interface that can set and manage alerts and display snapshots or graphs of monitored element. Network monitoring software is a system administration tool, not a security tool like the intrusion detection systems (IDS) or the intrusion prevention systems (IPS).

\subsection{Agent system}

In retrospect, the challenge of lack of standards faced by agent-based technology, they suffer from different terminology and heterogeneity of technical methods. Furthermore, the broad application of agent technologies to specific environments such as network management was still in infancy; (Bohoris, 2003). Software agents are often more clearly understood through their attributes and behaviors. It is commonly agreed among researchers that every agent exhibit several (but rarely all) of the following characteristics; (Wooldrige, 1995).

I. Proactive/active: often agents are required to anticipate future situations along with responding to changes in their environment.

II. Communicative/collaborative: no agent has a complete picture of the overall system which it operates. Each agent is an expert in a specific task and it has to collaborate with other agents in order to solve a given problem

\section{RESEARCH METHODOLOGY}

Prometheus methodology was used in the system specification, its design and implementation. The Prometheus methodology is a detailed process for specifying, designing and implementing intelligent agent system. Our goal in using Prometheus methodology was to have a process design with defined milestones. Prometheus is also amenable to tool support and provide scope for cross checking between designs, it was preferred over other methodologies because it has detailed and elaborates process for system specification, implementation, etc.

\subsection{Reasons for Prometheus methodology}

Prometheus methodology distinguishes itself from other methodologies by supporting the development of agent-based technology i.e. intelligent agents. It provides "start to end" support, having evolved out of the practical industry and having been used in industry and academia and above all, in being detailed and complete.

\subsection{Analysis using Prometheus concepts}

The analysis of this system was done using multi-agent system development known as PROMETHEUS which was used for designing intelligent software agents and agent systems. The methodology provides detailed guidance in terms of processes as well as notations. The analysis models for the system are represented using the concept and symbols for PROMETHEUS methodology.

\subsection{Related works}

Akuku (2011) carried out a study on Agent based audit monitoring system which utilizes agent properties and providing ability to monitor local or remote activities and events within the database with real time alerting and notification. The purpose was to develop a proactive database auditing system using multiagent technology that provide real time audit reports and alerts to auditors, independent of the database system. 
Carvalho et al., (2012) proposed a secured multi-agent based system in which different mobile agents were created for the purpose of getting network related information. Mobile agents retrieve network related information, which act as an input for network administrator. It keeps an eye on an activity of all registered clients using the mobile agent. The proposed application also focuses on reducing the network bandwidth used for monitoring the network by using agent per client architecture (mobile/roaming agent).

\subsection{System architecture}

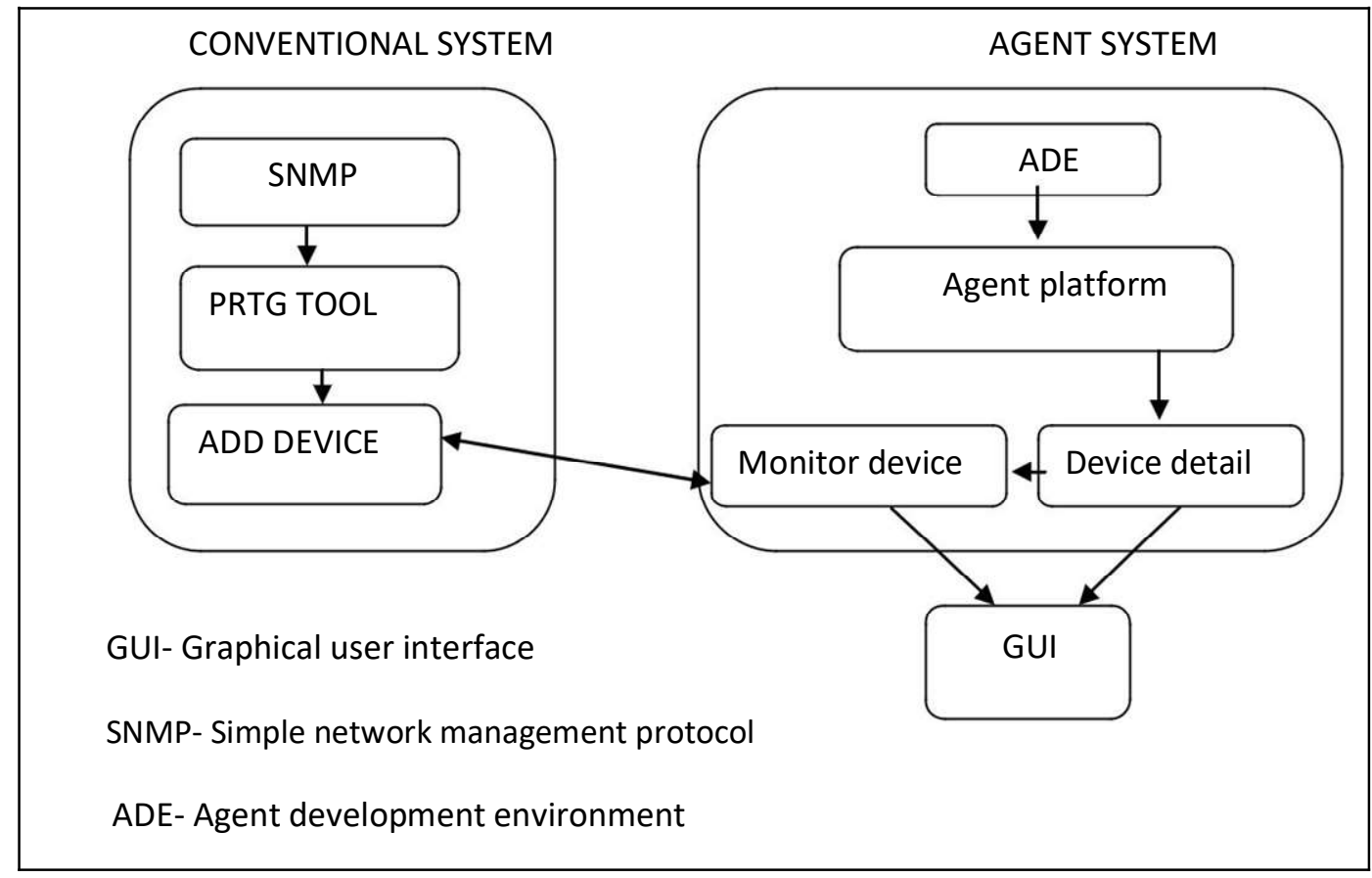

Fig. 1: Proposed System Arhitecture 


\section{IMPLEMENTATION/RESULTS Implementation tools}

Netbeans is an integrated development environment (IDE) for developing primarily with Java, but also with other languages in particular PHP, C/C++, and HTML5. It is also an application platform framework for Java desktop applications and others. The NetBeans IDE is written in Java and can run on Windows, OS X, Linux, Solaris and other platforms supporting a compatible JVM.

JADE framework: JADE (Java Agent Development Framework) is a software framework fully implemented in Java language. It simplifies the implementation of multi-agent systems through a middle-ware that claims to comply with the FIPA specifications and through a set of tools that supports the debugging and deployment phase.

Java JDK: This is an implementation of either one of the Java SE, Java EE or Java ME platforms released by Oracle Corporation in the form of a binary product aimed at Java developers on Solaris, Linux, Mac OS X or Windows. Since the introduction of the Java platform, it has been by far the most widely used Software Development Kit (SDK).

JAVA JRE: This is a Run Time Environment for Java applications.

PRTG network software: PRTG network monitor is the powerful network monitoring solution from Paessler AG. It ensures the availability of network components while also measuring traffic and usage. It saves costs by avoiding outages, optimizing connections and saving time.

\subsection{Minimum system requirements}

1. Hardware requirements
a. Window system (operating system32bit)
b. 10 Giga byte hard disk drive
c. 1 Gig Random Access Memory (size)
d. keyboard
e. color monitor

2. Software requirements
a. Netbeans IDE
b. JADE framework
c. Apache server
d. PRTG network tool
e. JRE runtime 
4.2 Outcome/result of the implementation

System evaluation was carried out to ensure that the system meets the user requirements and 6 the No. $^{4}$ Dec, 2018 specification. After evaluation, the system was able to achieve the following:

1. System administrator was able to interact with the system using the GUI interface

2. Agents/monitoring tool were able to pick necessary details from a client (workstation) and use for monitoring

3. The results were presented in graphical format

4. Uptime and down time status of a client are indicated on a GUI interface

5. Memory usage of a client is indicated in a GUI interface

6. CPU load of a client is displayed in GUI interface

7. Comparison of the conventional system against the agent based system using a bar chart

Table 1: Development Testing

\begin{tabular}{|c|c|c|c|c|c|}
\hline $\mathrm{S} / \mathrm{N}$ & Component Tested & Purpose & Condition & Test Data & Result \\
\hline 1 & User Authentication & $\begin{array}{l}\text { Correct login } \\
\text { to display GUI }\end{array}$ & Login GUI & $\begin{array}{l}\text { Login name } \\
\text { and password }\end{array}$ & $\begin{array}{l}\text { Main menu } \\
\text { display }\end{array}$ \\
\hline 2 & New client creation & $\begin{array}{l}\text { Successful } \\
\text { creation of } \\
\text { new client }\end{array}$ & $\begin{array}{l}\text { client Data } \\
\text { GUI }\end{array}$ & $\begin{array}{l}\text { IP address, IP } \\
\text { version }\end{array}$ & $\begin{array}{l}\text { Creation of } \\
\text { new client }\end{array}$ \\
\hline 3 & Uptime status & $\begin{array}{l}\text { View uptime } \\
\text { statistic of a } \\
\text { client }\end{array}$ & $\begin{array}{l}\text { client Data } \\
\text { GUI }\end{array}$ & IP address & $\begin{array}{l}\text { Uptime } \\
\text { statistic }\end{array}$ \\
\hline 4 & Memory Usage & $\begin{array}{l}\text { View memory } \\
\text { usage of a } \\
\text { client }\end{array}$ & $\begin{array}{l}\text { client Data } \\
\text { GUI }\end{array}$ & IP address & $\begin{array}{l}\text { Memory } \\
\text { Usage (graph) }\end{array}$ \\
\hline 5 & CPU load & $\begin{array}{l}\text { View CPU } \\
\text { load of a client }\end{array}$ & $\begin{array}{l}\text { client data } \\
\text { GUI }\end{array}$ & IP address & CPU usage \\
\hline
\end{tabular}


COMPARISON OF AGENT BASED NETWORK MONITORING AGAINST SNMP

Table 2: Results

\begin{tabular}{|l|cc|c|c|}
\hline PARAMETERS & $\begin{array}{l}\text { Average } \\
\text { initiate } \\
\text { SNMP }\end{array}$ & AGENT & $\begin{array}{l}\text { time to } \\
\text { (PARAMETERS) }\end{array}$ & OS REQUIRED \\
\hline & 10 & 5 & GUI & WINDOW \\
uptime status & 8 & 3 & GUI & WINDOW \\
memory usage & 10 & 4 & GUI & WINDOW \\
CPU load & 7 & 5 & GUI & WINDOW \\
hard disk volume & 6 & 3 & GUI & WINDOW \\
device health & 5 & 2 & GUI & WINDOW \\
WIFI traffic & & & & \\
\hline
\end{tabular}

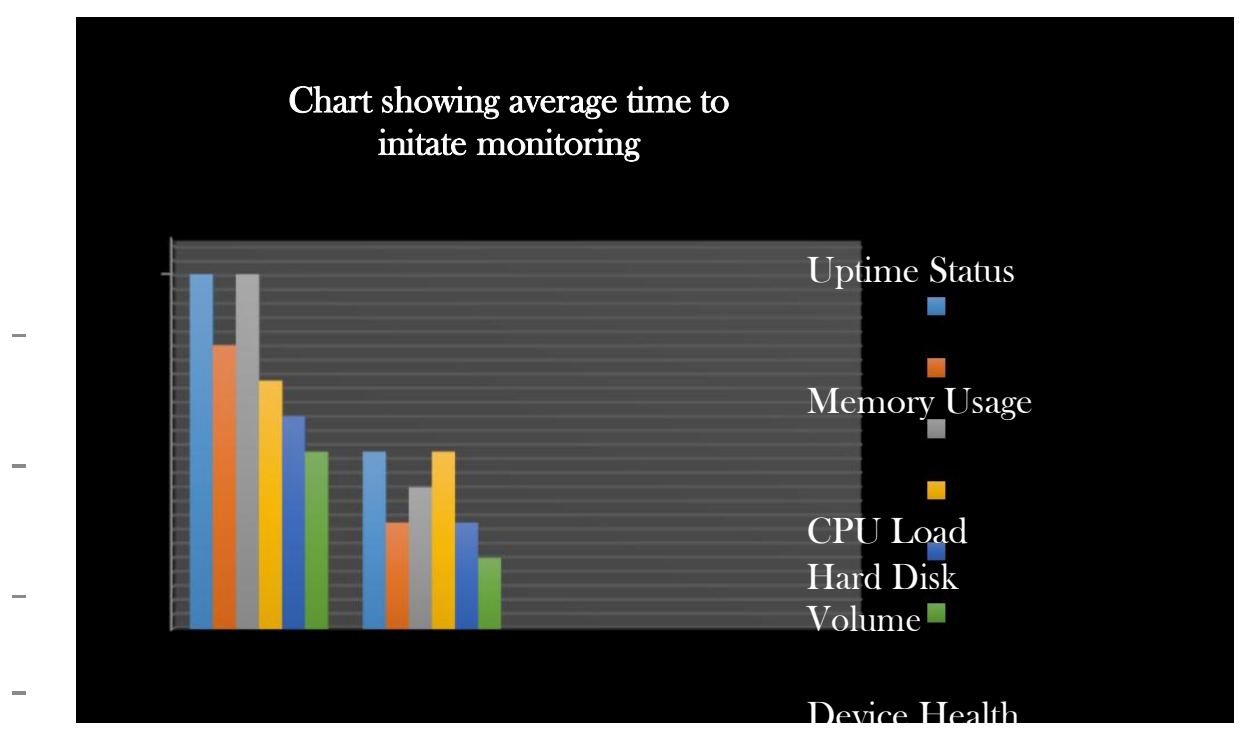

Fig 2: Average Time Chart 


\section{CONCLUSION}

Network management (monitoring) is very effective in today's life as it is important in businesses and many large organization. The analysis and design of network monitoring application using agent model is highly efficient as it enhances the conventional or existing system of network monitoring (network management). With the introduction of the agent based technology, it reduces the time it takes to initiate monitoring on any network node.

This research paper has shown that agent/multi-agents can be used to provide more responsive monitoring solutions on network nodes. Mobile agents offer a flexible and scalable solution to network monitoring in the management of today's complex and heterogeneous communication networks, hence reduces the number of unnecessary human interactions. The autonomy and mobility of mobile agents reduces bandwidth overloading problems by moving processing and decision making from centralized network management stations over to the managed devices, thus using agents network monitoring and other management tasks can be easily decentralized.

\section{REFERENCES}

1. Akuku, B. (2011). Agent Based System for Real Time Database Audit Monitoring: Nairobi: University of Nairobi.

2. Bohoris, C. (2003). Mobile Agent-Based Performance Management for the Virtual Home Environment: In Journal of Network and System Management, 11(2), 133-149.

3. Carvalho L., Nielet D'. (2013). Secure Network Monitoring System Using Mobile Agents International Journal of Modern Engineering Research, 3(3), 1850-1853.

4. Jacques, F. (1999). Multi-Agent System: An Introduction to Artificial Intelligence, Addison Wesley.

5. Wesley, A. (1999). Practical Network Management: SNMP, SNMPv2 and RMON.

6. Wooldridge, M. and N. R. Jennings (1995). Intelligent Agents: Theory and Practical. In Knowledge Engineering Review, 10: 115 - 152.

7. Wooldridge, M. (2012). An Introduction to Multi-Agents Systems: John Wiley and Sons Limited England. 